\title{
ASYMPTOMATIC LABIAL FUSION DIAGNOSED DURING LABOUR -(A CASE REPORT)
}

\section{Dr. Anshu Baser \\ Dr. Bhoomika Jain*}

Dr. Sushil Kumar
MBBS, Junior Resident, Department of Obstetrics and Gynecology, MGM Medical College, Sector 4E, Kalamboli, Navi Mumbai, 410218

MBBS, Junior Resident, Department of Obstetrics and Gynecology, MGM Medical College, Navi Mumbai- 410218. *Corresponding Author 410218.

ABSTRACT Labial fusion is when the labia minora of the vulva fuse together. This most commonly occurs in prepubertal age group $(0.6 \%-5 \%)$.(1) This condition rarely occurs in adults and even in adults it is seen in post menopausal women and may result in urinary incontinence.(2) It is extremely rare in women of reproductive age group and only a few cases have been reported so far. (3) We here report a case of a 25 year old female with asymptomatic labial fusion with no history of no history of voiding difficulty or dyspareunia, who presented to us at term in labour.

\section{KEYWORDS : Labial fusion in pregnancy, labial adhesion, conception with labial fusion.}

\section{INTRODUCTION:}

Labial fusion or adhesions is defined as partial or complete fusion of the labia minora. Labial fusion is usually seen in prepubertal age group $(0.6 \%-5 \%)(1)$. It usually occurs between 3 months and 3 years.(4) It is extremely uncommon in adult population and only a few cases have been reported so far. (3)

The etiology of labial fusion is believed to be due to vaginal inflammation or irritation, and is associated with low oestrogen status.(4) However no concrete evidence has been found regarding the same. Labial fusion can lead to post-void dripping (urinary incontinence), urinary tract infections, vaginitis (vaginal irritation), hematuria and urinary frequency. (5) Vaginal inflammation-irritation, hypoestrogenism, local trauma, lack of sexual activity, female circumcision, vaginal lacerations in childbirth and recurrent urinary tract infections are formerly reported as the contributing factors for LF in adults especially in postmenopausal women.(6)

Here we describe a case of asymptomatic labial fusion in a 25year old female who conceived spontaneously, went up to term and then in spontaneous labour.

\section{Case Report:}

A 25 year old female with 9 months of amenorrhoea presented to us with the complaints of pain in abdomen and bleeding per vaginum. On examination her general condition was fair and vitals were stable On Per abdomen examination she was full term with cephalic presentation with regular fetal heart sounds and she had active uterine contractions. On local examination the labia minora was fused.(Fig 1) There was bleeding present per vaginum. The introitus was small and on examination with a small size retractor, there were multiple vaginal lacerations seen and a tiny portion of fetal head was visible. The urethra was seen below the fused labia minora. (Fig 2) However the patient had regular menstrual cycles. She gave no history of urinary incontinence or voiding difficulty, there was also no history of dyspareunia, difficult intercourse and had spontaneously conceived within 1 year of marriage. She also did not give any history of any genital injury/ operation (genital mutilation) carried out at birth. Patient delivered via emergency caesarean section a heathy female baby. The vaginal lacerations were sutured post delivery. An ultrasound was also done post delivery to rule out any renal or Mullerian anomalies which did no reveal any significant findings. No immediate intervention was undertaken for the labial fusion. The patient was discharged on post operative day 5 with advice to follow up after one week.

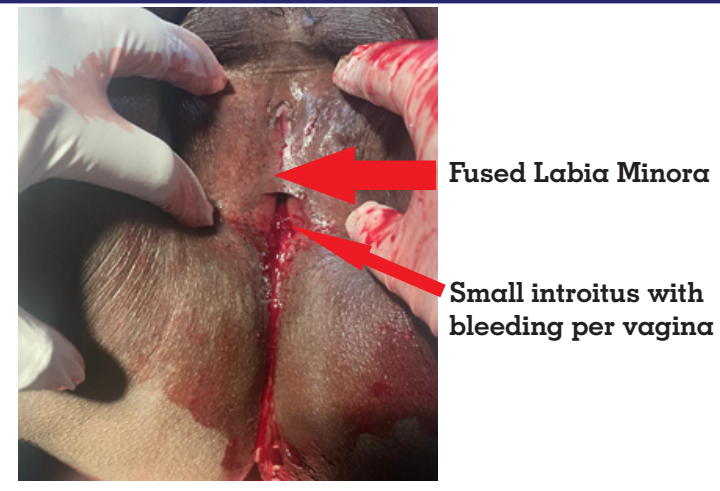

Fig 1 Showing Fusion Of Labia Minora

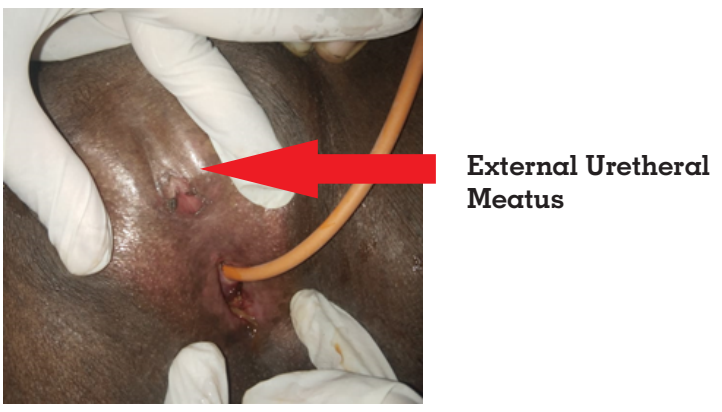

Fig 2 Showing Urethral Meatus Below The Fused Labia Minora

\section{DISCUSSION:}

Labial fusion or adhesions in reproductive age group is extremely rare. Adequate literature regarding presentation of the condition in young adults is not yet available. Majority of cases reported were asymptomatic. (3)

In some instances patients presented with urinary tract infections, urinary retention, vulvo-vaginitis genital trauma, female circumcision, hypoestrogenism and lack of sexual activity. Spontaneous conception with labial fusion is also rare only a handful of cases have been reported. Congenital labial fusion is a possibility here and is associated with use of drugs such as danazol. (7) It may also be associated with adrenal steroid 21-hydroxylase deficiency. (8)

Another association has also been seen with an autosomal recessive disorder Fraser syndrome. (9) 
Genital mutilation/ female circumcision when carried out in infancy in some cultures can also lead to genital scarring and fibrosis which may lead to labial fusion (10)

In our case the patient had regular menstrual cycles and regular intercourse prior to conception hence hypoestrogenism was not a factor. She had well developed secondary sexual characteristics and no history of any drug intake. There was no history of consanguinity or female circumcision. There were no other physical anomalies except external genitalia. Therefore possibility of congenital labial fusion cannot be ruled out.

The treatment options for labial fusion are topical oestrogen cream with or without manual separation. However these have only be evaluated in pediatric population. In a study conducted by Ellen et al in 71 pediatric patients it was found that the success rate was $35 \%$ for oestrogen and $80 \%$ for manual separation. (1 l)

Labial separation during pregnancy has not been adequately evaluated and only a few cases have been reported. In this case it was not done at the time of delivery as there was risk of severe bleeding due to the vulva being highly vascular in pregnancy. Patient was asymptomatic post delivery, she had no complaints of dyspareunia or urinary incontinence hence no medical or surgical intervention was undertaken for this patient.

\section{Summary}

labial fusion is extremely rare in adults and especially during pregnancy. We here report a rare case. A 25 year old female presented with asymptomatic labial fusion at full term in labour. Patient delivered a healthy female baby by emergency cesarean section. No intervention was undertaken at the time. Patient had no complications post delivery and no complaints of sexual dysfunction or urinary incontinence.

\section{Conflict Of Interest And Funding:}

The Authors declare that there is no conflict of interest. No funding was obtained for this publication.

\section{Ethical Consideration:}

Approval for publication of this article was taken from institutional ethical committee. Patient consent was taken for publication of photographs and case details.

\section{REFERENCES:}

1) Mayoglou L, Dulabon L, Martin N and Schober J (2009) Success of treatment for labial fusion: A retrospective evaluation of topical and surgical treatments. J Pediatr Adolesc Gynecol 22: 247-250.

2) Mahran, M.A., Oligbo, N. Labial fusion causing urinary incontinence: comment. Int Urogynecol J 22, 1335 (2011). https://doi.org/10.1007/s00192011-1496-y

3) Al-Hubaishi F, Al-Najjar F, Al-Medfa AS (2018) A Case Report on an Asymptomatic Labial Fusion in a Woman of reproductive Age. Gynecol Obstet Case Rep Vol.4:No.1:60

4) TOPCUOGLU, M. A., KOC, O., DURAN, B., \& DONMEZ, M. (2009). Labial fusion causing acute urinary retention in a young adult: $A$ case report. Australian and New Zealand Journal of Obstetrics and Gynaecology, 49(1), 115-116.

5) Murat Ozekinci, Selcuk Yucel, Cem Sanhal, Munire Erman Akar Labial Fusion Causing Coital and Voiding Difficulty in a Young Woman. Advances in Sexual Medicine;Vol.3 No.1(2013), Article ID:27128,3 pages

6) A. Dirim and E. Hasirci, "Labial Fusion Causing Urinary Incontinance and Recurrent Urinary Tract Infection in a Postmenopausal Female: A Case Report," International Urogynecology Journal, Vol. 22, No. 1, 2011, pp. 119-120.

7) Dolezalkova E, Matura D, Simetka O. A case report of congenital labial fusion. 14th World Congress in Fetal Medicine.

8) Aziz Siddiqui S, Soomro N, Ganatra A. Classic congenital adrenal hyperplasia: A delayed presentation. PakJ Med Sci. 2013;29(1):220-223.

9) Mbonda, A., Endomba, F.T., Kanmounye, U.S. et al. Diagnosis of Fraser syndrome missed out until the age of six months old in a low-resource setting: a case report. BMC Pediatr 19, 292 (2019).

10) Horowitz, C. R., \& Jackson, J. C. (1997). Female "circumcision" African women confront American medicine. Journal of General Internal Medicine, 12(8), $491-499$

11) Wejde, E., Ekmark, A. N., \& Stenström, P. (2018). Treatment with oestrogen or manual separation for labial adhesions - initial outcome and long-term follow-up. BMC Pediatrics, 18(1). 